\title{
Reactive Interstitial and Reparative Fibrosis as Substrates for Cardiac Ectopic Pacemakers and Reentries
}

\author{
Rafael Sachetto Oliveira ${ }^{1}$, Bruno Gouvêa de Barros ${ }^{2}$, Johnny Moreira Gomes ${ }^{2}$, \\ Marcelo Lobosco ${ }^{2}$, Sergio Alonso ${ }^{3}$, Markus Bär ${ }^{4}$, and \\ Rodrigo Weber dos Santos ${ }^{2}$ \\ 1 Departamento de Ciência da Computação, Universidade Federal de São João del \\ Rei, São João del Rei, Brazil \\ 2 Departamento de Ciência da Computação e Programa em Modelagem \\ Computacional, Universidade Federal de Juiz de Fora, Juiz de Fora, Brazil \\ 3 Department of Physics, Universitat Politècnica de Catalunya, Barcelona, Spain \\ ${ }^{4}$ Physikalisch-Technische Bundesanstalt, Braunschweig and Berlin, Germany
}

\begin{abstract}
Dangerous cardiac arrhythmias have been frequently associated with focal sources of fast pulses, i.e. ectopic pacemakers. However, there is a lack of experimental evidences that could explain how ectopic pacemakers could be formed in cardiac tissue. In recent studies, we have proposed a new theory for the genesis of ectopic pacemakers in pathological cardiac tissues: reentry inside microfibrosis, i.e., a small region where excitable myocytes and non-conductive material coexist. In this work, we continue this investigation by comparing different types of fibrosis, reparative and reactive interstitial fibrosis. We use detailed and modern models of cardiac electrophysiology that account for the micro-structure of cardiac tissue. In addition, for the solution of our models we use, for the first time, a new numerical algorithm based on the Uniformization method. Our simulation results suggest that both types of fibrosis can support reentries, and therefore can generate in-silico ectopic pacemakers. However, the probability of reentries differs quantitatively for the different types of fibrosis. In addition, the new Uniformization method yields 20 -fold increase in cardiac tissue simulation speed and, therefore, was an essential technique that allowed the execution of over a thousand of simulations.
\end{abstract}

\section{Introduction}

The function of the heart, in physiological conditions or pathological ones, is a non-linear, multi-scale and multi-physics phenomenon, and as such, poses incredible challenges to science and medicine. Computational and mathematical models of the heart have supported and complemented experimental techniques proposing answers to both basic and applied questions regarding cardiac physiology. In particular, in the area of cardiac electrophysiology, for decades, models have been used to propose and test new theories for basic mechanisms [4,2], during the tests of drugs [20] and development of new medical devices [8]. 
Dangerous cardiac arrhythmias, such as atrial and ventricular fibrillation have been frequently associated to fibrosis $[26,9,14,24]$. For instance, during atrial fibrillation the duration of fibrillation episodes was highly correlated to the degree of fibrosis, i.e. the amount of fibrous tissue in the extracellular space between myocytes and/or fibrous tissue that replaced dead myocytes [16]. Nevertheless, in the aforementioned works, whether in animal or computational experiments, an artificial trigger is usually used to induce the arrhythmia, a focal stimulation site that induces rapid pacing. This focal trigger is often called ectopic pacemaker.

Unfortunately, the mechanisms and conditions for the formation of a region that becomes an ectopic pacemaker are until now poorly understood. Some new evidences suggest that two mechanisms (combined or not) may play a very important role in the generation of such dangerous triggers of arrhythmia: abnormal intracellular calcium $(\mathrm{Ca} 2+)$ signaling $[25]$ and microreentry (reentry inside a fibrotic region).

In our previous works $[1,6]$ we were able to generate an ectopic pacemaker in computer simulations of cardiac electrophysiology by assuming a single hypothesis for substrate: the existence of a region of microfibrosis, a mixture of excitable (healthy myocytes) and non-conducting (fibrosis) areas. The mechanism of this ectopic pacemaker is simple. The two different phases (excitable and nonconducting) form a maze for wave propagation. Propagation fractionates and follows zig-zag pathways. Macroscopic propagation is considerably slowed down. Microscopically, the topology of the maze allows the electric wave to reexcite the fibrotic region before the wave leaves it. Therefore, reentry occurs inside the fibrotic region. This spiral-like wave will continuously try to generate ectopic beats when it touches the border of the microfibrosis region [12].

Here, we continue this investigation by comparing different types of fibrosis, reparative and reactive interstitial fibrosis. The excessive deposition of collagen in the extracellular matrix may physically and electrically separate neighboring myocytes, a process called reactive interstitial fibrosis, or completely replace a dead myocyte, a process called reparative fibrosis [21].

Our computational models are based on the microscopic tissue model that uses subcellular discretization of $8 \mu \mathrm{m}$ and detailed and realistic gap junction distribution [5]. From this basic microscopic model we generate an equivalent discrete model [6]. With the discrete or network-like model we simulated reactive interstitial fibrosis by randomly removing a certain percentage of links between neighboring cells; and simulate reparative fibrosis by randomly removing a certain percentage of myocytes from the network.

Since our models account for realistic description of single myocyte electrophysiology and for the microstructure of cardiac tissue such as gap junction distribution and tissue anisotropy, they are computationally very expensive to solve. To speed up the simulations, we use in this work the new Uniformization method, first proposed and tested for single cell simulations [15]. This is the first time this new numerical method is tested in the framework of simulations of cardiac tissue. 


\section{Methods}

\subsection{Modeling Fibrosis}

We use a microscopic tissue model with subcellular discretization of $8 \mu \mathrm{m}$ to represent detailed shapes of myocytes as well as realistic gap junction distribution [5] and presented in the top of Figure 1. From this basic microscopic model we generate an equivalent discrete model [6] and presented in the bottom of Figure 1. With the discrete or network-like model we simulated reactive interstitial fibrosis by randomly removing a certain percentage of links between neighboring cells, $\phi_{l}$ (see Figure 2); and to simulate reparative fibrosis we randomly removed a certain percentage of cells, $\phi_{c}$, from the network (see Figure 1).

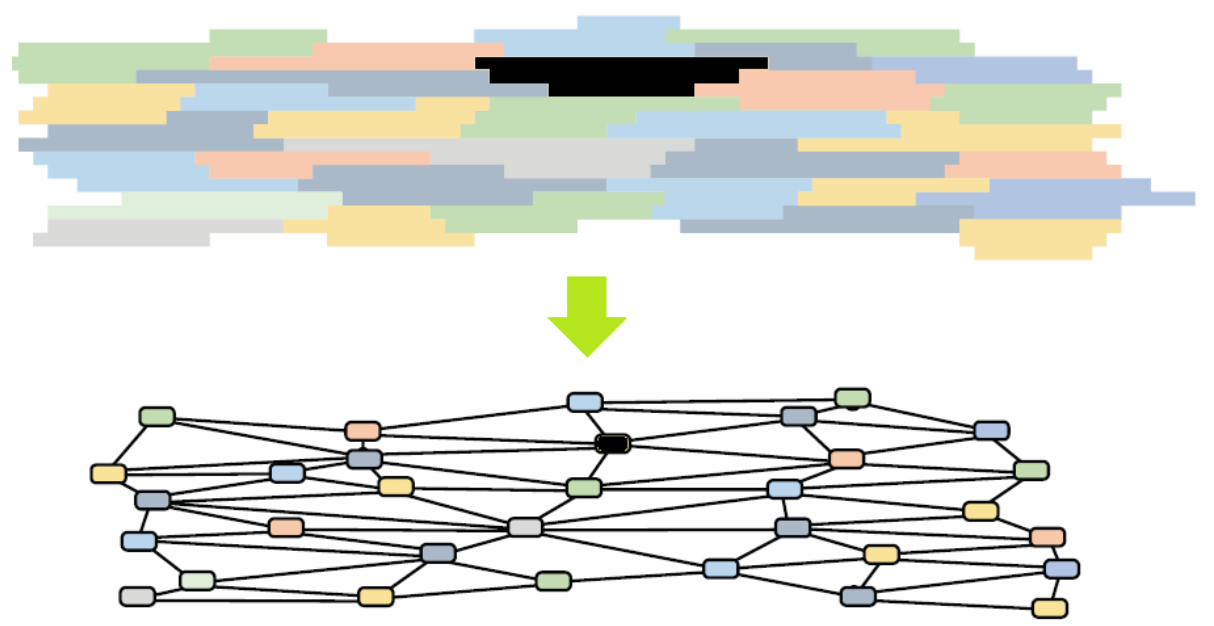

Fig. 1: Basic microscopic model with subcellular discretization to represent detailed shapes of myocytes as well as realistic gap junction distribution (Top) and the equivalent detailed discrete model (Bottom) where each myocyte is represented by one cell in the network. A black shaded myocyte represents how reparative fibrosis is modeled in the microscopic model (Top) and in the equivalent detailed discrete model (Bottom).

The purpose of our work is to assess the probability of a certain fibrotic tissue to become an ectopic pacemaker and to study how it depends on the fraction and type of fibrosis, $\phi_{c}$ and $\phi_{l}$. To achieve this goal it is necessary to perform thousands of simulations of different microfibrosis models.

For instance, to simulated reparative fibrosis, for each given value of $\phi_{c}$ we randomly generate a microfibrosis tissue. Fifty realizations of different microfibrosis but with the same value for $\phi_{c}$ were simulated. This process is represented 
by Figure 3. The value of $\phi_{c}$ was varied from $44 \%$ to $64 \%$, in steps of $2 \%$. Therefore a total of $11 \times 50=550$ cardiac tissues with different characteristics of reparative fibrosis were simulated. The same was performed for the case of reactive interstitial fibrosis, i.e. the value of $\phi_{l}$ was also varied from $44 \%$ to $64 \%$, in steps of $2 \%$. Again a total of $11 \times 50=550$ cardiac tissues with different characteristics of reactive interstitial fibrosis were simulated. For all the results presented in the next section the modeled cardiac tissues had dimensions of 1 $\mathrm{cm} \times 1 \mathrm{~cm}$.

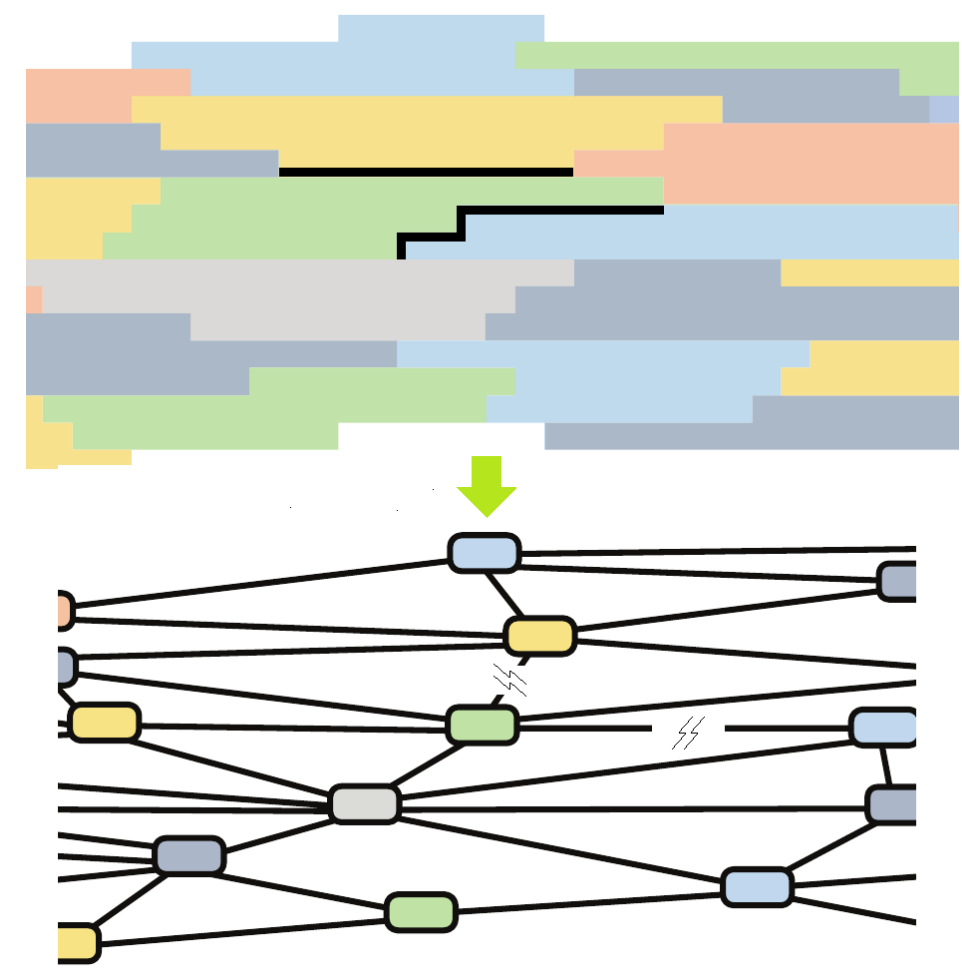

Fig. 2: Black shaded areas represent how reactive interstitial fibrosis is modeled in the microscopic model (Top) and in the equivalent detailed discrete model (Bottom).

\subsection{Numerical Methods}

The detailed and discrete model of cardiac tissue was numerically solved [6] using the finite volume method. The reaction and diffusion parts of the discrete monodomain equations were split by employing the Godunov operator splitting 

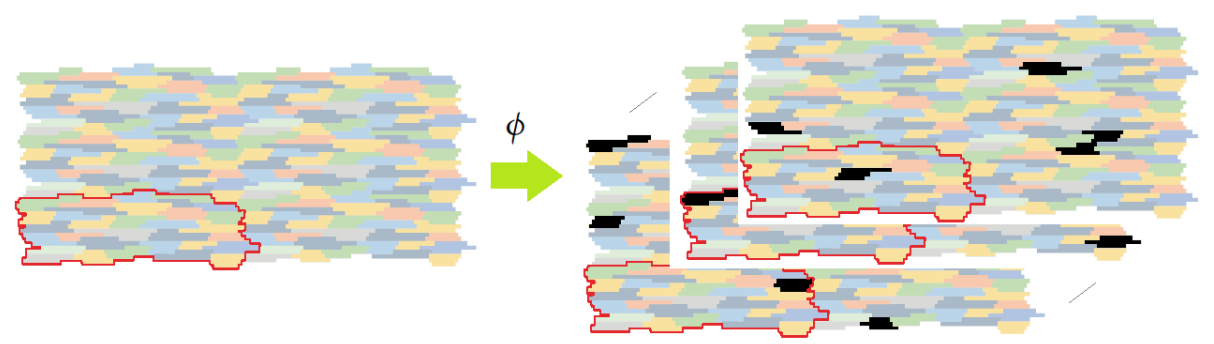

Fig. 3: Multiple instances of models generated with the same percentage of reparative fibrosis, $\phi_{c}$. The size of these illustrative cardiac tissue models is $1.1 \mathrm{~mm} \times 0.4 \mathrm{~mm}$.

[22]. Therefore, each time step involves the solution of two different problems: a nonlinear system of ODEs

$$
\begin{aligned}
\frac{\partial V_{i}}{\partial t} & =\frac{1}{C_{m}}\left[-I_{i o n}\left(V_{i}, \boldsymbol{\eta}_{\boldsymbol{i}}\right)+I_{s t i m}\right] \\
\frac{\partial \boldsymbol{\eta}_{\boldsymbol{i}}}{\partial t} & =f\left(V_{i}, \boldsymbol{\eta}_{\boldsymbol{i}}\right)
\end{aligned}
$$

and a linear system from the discrete monodomain equation

$$
\operatorname{Vol}_{i} \beta\left(C_{m} \frac{\partial V_{i}}{\partial t}+I_{i o n}\left(V_{i}, \boldsymbol{\eta}_{i}\right)\right)=\left(\sum_{j=1}^{n n_{i}}\left(G_{i, j} *\left(V_{j}-V_{i}\right)\right)+I_{i}^{\text {stim }},\right.
$$

for each node $i$ of the discrete topology, where $G_{i, j}$ is the equivalent conductance between cell $i$ and cell $j$. Each cell has different geometry $\left(V o l_{i}\right)$ and a different number of neighboring cells $\left(n n_{i}\right) . V$ is the variable of interest and represents the transmembrane voltage, i.e. the difference between intracellular to extracellular potential; $\boldsymbol{\eta}$ is a vector of state variables that also influence the generation and propagation of the electric wave, and usually includes the intracellular concentration of different ions $\left(\mathrm{K}^{+}, \mathrm{Na}^{+}, \mathrm{Ca}^{2+}\right)$ and the permeability of different membrane ion channels; $\beta$ is the surface-volume ratio of heart cells; $C_{m}$ is the membrane capacitance, $I_{i o n}$ the total ionic current, which is a function of $V$ and a vector of state variables $\boldsymbol{\eta}, I_{\text {stim }}$ is the current due to an external stimulus. We assume that the boundary of the tissue is isolated, i.e., no-flux boundary conditions were imposed.

For the discretization of the nonlinear system of ODEs we note that its stiffness demands very small time steps. For simple models based on HodgkinHuxley formulation this problem is normally overcome by using the Rush-Larsen (RL) method [18]. However, for the most modern and complex models that are highly based on Markov Chains, the RL method seems to be ineffective in terms of allowing larger time steps during the numerical integration.

In this work we use the modern Markov-based model of Bondarenko et al. [7] for myocyte electrophysiology. For the case of the Bondarenko et al. model, 
we tested both methods, Euler and RL, and both demanded the same time step, $\Delta t_{o}=0.0001 \mathrm{~ms}$ due to stability issues. Since the RL method is more expensive per time step than the Euler method, in this work, we used either the simple explicit Euler method for the discretization of the nonlinear ODEs or the new Uniformization and SAST1 method [15].

For the discrete monodomain equation we used the unconditionally stable implicit Euler scheme. This allowed us to use longer time steps for the numerical solution of it than those used for the solution of ODEs $\left(\Delta t_{p}>\Delta t_{o}\right)$. This linear system was solved in parallel with the PETSc library [3]. The nonlinear system of ODEs were also solved in parallel using the MPI library [10].

In the next sections we give more details about the new numerical methods of SAST1 and Uniformization.

The Extended Rush-Larsen Method (SAST1) The method proposed by Rush-Larsen (RL) [19] is very popular in the community of cardiac electrophysiology and focus on the gating variables from Hodgkin and Huxley's formulation [11]. This method is based on a local linearization of the equations in the form

$$
\frac{d y}{d t}=\alpha(V)(1-y)-\beta(V) y,
$$

so the equations associated with the gating variables are numerically solved by:

$$
\begin{aligned}
y_{n+1} & =\left(y_{n}-\frac{\alpha}{\alpha+\beta}\right) e^{-(\alpha+\beta) h}+\frac{\alpha}{\alpha+\beta}, \\
k & =|\alpha+\beta|,
\end{aligned}
$$

where $h$ is the time step. The remaining equations of the model are evaluated by the Euler method. Therefore, this is a first order method. If the value of $k$, defined by equation (6), is close to zero we use the Euler method instead of equations (5) for the corresponding gating variable at that point of the simulation.

The first order version of Sundnes et al. method (SAST1) [23] extends the RL method for each and every differential equation of the model. This is achieved after the following linearization around $y^{j}\left(t_{n}\right)$, where $t_{n}=n h$ and $h$ is the time step:

$$
\begin{aligned}
\frac{d y^{j}(t)}{d t} & =f^{j}\left(\vec{Y}\left(t_{n}\right), t_{n}\right)+k\left(y^{j}(t)-y^{j}\left(t_{n}\right)\right), \\
k & =\frac{\partial f^{j}\left(\vec{Y}\left(t_{n}\right), t_{n}\right)}{\partial y^{j}},
\end{aligned}
$$

whereas $\vec{Y}$ is the vector containing the variables of the model $y^{j}$ as components and $f^{j}$ is the right-hand side function for variable $y^{j}$. This can now also be considered a quasi-linear equation with an analytic solution similar to the RL one. 
The SAST1 method is presented in the next equations, where the partial derivative is approximated via finite differentiation:

$$
\begin{aligned}
y_{n+1}^{j} & =y_{n}^{j}+\frac{f^{j}\left(\overrightarrow{Y_{n}}, t_{n}\right)}{k}\left(e^{h k}-1\right), \\
k & =\frac{\partial f^{j}\left(\overrightarrow{Y_{n}}, t_{n}\right)}{\partial y^{j}} .
\end{aligned}
$$

Uniformization Method The Uniformization (or randomization) method [13] is an efficient technique for the transient analysis of Markov models. The method is traditionally used for reliability, dependability and performance analysis of computer systems modeled by Markov models.

Let $\pi(t)$ be the probability line vector of a continuous time Markov Chain (CTMC) and $\mathbf{P}(t)$ the associated transition matrix, whereas $[\mathbf{P}]_{i j}$ is the transition rate from state $i$ to state $j$. So the ODEs system associated with the CTMC is given by $\frac{d \pi}{d t}=\pi \mathbf{P}$. If we consider the terms $[\mathbf{P}]_{i j}$ as constant in a small time interval, the ODEs system will be locally linear. Therefore, the solution of the system within this small time interval is given by $\pi(t+h)=\pi(t) e^{h \mathbf{P}}$, whereas the matrix exponential is defined by the Taylor series as $e^{t \mathbf{P}}=\sum_{i=0}^{\infty} \frac{(t \mathbf{P})^{i}}{i !}$.

The direct usage of matrix $\mathbf{P}$ leads to numerical errors and slow convergence in the evaluation of the series. The main reasons for this are: (1) diagonal terms of $\mathbf{P}$ are negative and the remaining terms are non-negative and; (2) the existence of terms with magnitude greater than 1. The Uniformization method defines $\mathbf{P}^{*}=\mathbf{P} / q+I$, where $q \geq \max _{1 \leq i \leq N_{m k}}\left|[\mathbf{P}]_{i i}\right|$ and $N_{m k}$ is the number of states in the CTMC. Since $\mathbf{P}=q\left(\mathbf{P}^{*}-I\right)$, we have

$$
\pi(t+h)=\pi(t) e^{q t\left(\mathbf{P}^{*}-I\right)}=e^{-q t} \pi(t) e^{q t \mathbf{P}^{*}}
$$

so the resulting solution using the Taylor series is

$$
\pi(t+h)=e^{-q t} \pi(t) \sum_{i=0}^{\infty} \frac{\left(q t \mathbf{P}^{*}\right)^{i}}{i !}
$$

In order to truncate the series in Eq. (11) we can use the following relation [17] to choose the number of evaluated terms $N$, given an error tolerance $\lambda$ :

$$
\lambda \leq 1-e^{-q(t) h} \sum_{i=0}^{N} \frac{(q(t) h)^{i}}{i !} .
$$

In this paper, the implementation called $U n i+S A S T 1$ uses the Uniformization method for all the equations modeled by Markov Chains and uses the SAST1 method for the remaining equations in the Bondarenko et al. cardiac electrophysiology model. 


\subsection{Computing Environment}

All the numerical experiments were performed using a GNU/Linux 2.6.32 machine, with 12GB of memory and two Intel Xeon E5620 2.40 GHz processors each with 4 cores and $12 \mathrm{MB}$ of cache memory. Our monodomain solver was implemented in $\mathrm{C}++$ using PETSc 3.5.1 [3] and MPI, and compiled using GNU GCC 4.4.7 with $-O 3$ optimization flag enabled.

\subsection{Computational Simulations}

In order to evaluate the performance of the Uniformization+SAST1 method compared to the classical Euler method we executed five simulations using different values of $\phi_{c}$ to randomly remove a certain percentage of cells and generate reentry (values between 0.44 and 0.46 ). To analyze if the generated reentries would impact on the performance the Uni+SAST1 method, we fixed seeds in our random generation routine to ensure four simulations with reentry and one without, see section 3.2 for details about the reentries.

The simulations were performed using the detailed discrete model and a cardiac tissue of $1.0 \mathrm{~cm} \times 1.0 \mathrm{~cm}$ size and were executed for $500 \mathrm{~ms}$. The values used for $\beta$ and $C_{m}$ were set to $0.14 \mathrm{~cm}^{-1}$ and $1.0 \mu \mathrm{F} / \mathrm{cm}^{2}$, respectively. The time step used to solve the linear system associated to the discrete monodomain equation (PDE) was set to $\Delta t_{p}=0.02 \mathrm{~ms}$ and to solve the nonlinear system of ODEs associated was set to $\Delta t_{o}=0.0001 \mathrm{~ms}$ for the Euler method and 0.02 for the Uni+SAST1.

The same parameters were used to assess the probability of a certain fibrotic tissue to become an ectopic pacemaker and to study how this depends on the fraction and type of fibrosis, $\phi_{c}$ and $\phi_{l}$. The value of $\phi_{c}$, for the case of reparative fibrosis, was varied from $44 \%$ to $64 \%$, in steps of $2 \%$. The value of $\phi_{l}$, for the case of reactive interstitial fibrosis, was also varied from $44 \%$ to $64 \%$, in steps of $2 \%$. Cardiac tissues of $1.0 \mathrm{~cm} \times 1.0 \mathrm{~cm}$ size were executed for $200 \mathrm{~ms}$.

\section{Results}

\subsection{Uniformization vs. Euler}

Table 1 presents the execution times for the resolution of the PDE, ODEs and full simulation for both numerical methods. The time is an average of all different simulations using Euler or Uniformization and SAST1 (Uni+SAST1). The coefficients of variation (CV) for the Uniformization simulations were $3.71 \%$ for the PDE time, $0.9 \%$ for the ODEs and $2.3 \%$ for the Total time.

Analyzing the results in Table 1, we can see that the ODE time dominates the execution time, being $85 \%$ when using the Euler method. We can also see that, by using the Uni+SAST1 method, we were able to solve the ODEs $47 \times$ faster than using the Euler method. For the simulation total time, the speedup achieved was $19 \times$. 
Table 1: Execution times (average) for the resolution of the PDE, ODEs and full simulation for both numerical methods.

\begin{tabular}{rccc}
\hline Method & PDE $(\mathrm{s})$ & ODE $(\mathrm{s})$ & Total $(\mathrm{s})$ \\
\hline Euler & 3731.32 & 109659.13 & 129682.20 \\
Uni+SAST1 & 3594.73 & 2321.17 & 6839.28 \\
\hline
\end{tabular}

\subsection{Reactive Interstitial Fibrosis vs. Reparative Fibrosis}

As in our previous works $[1,6]$ we were able to generate reentry inside fibrotic tissues. Figure 4 shows color maps of the transmembrane voltage at four different instants along one of the simulations. The tissue was stimulated in the left border, the traveling wave fractionates and its zig-zag pattern of propagation supports a sustained reentry pattern of excitation.

Figure 5 presents the results in terms of probability of reentry for the two different types of fibrosis modeled in this paper: reparative vs. reactive intersti-
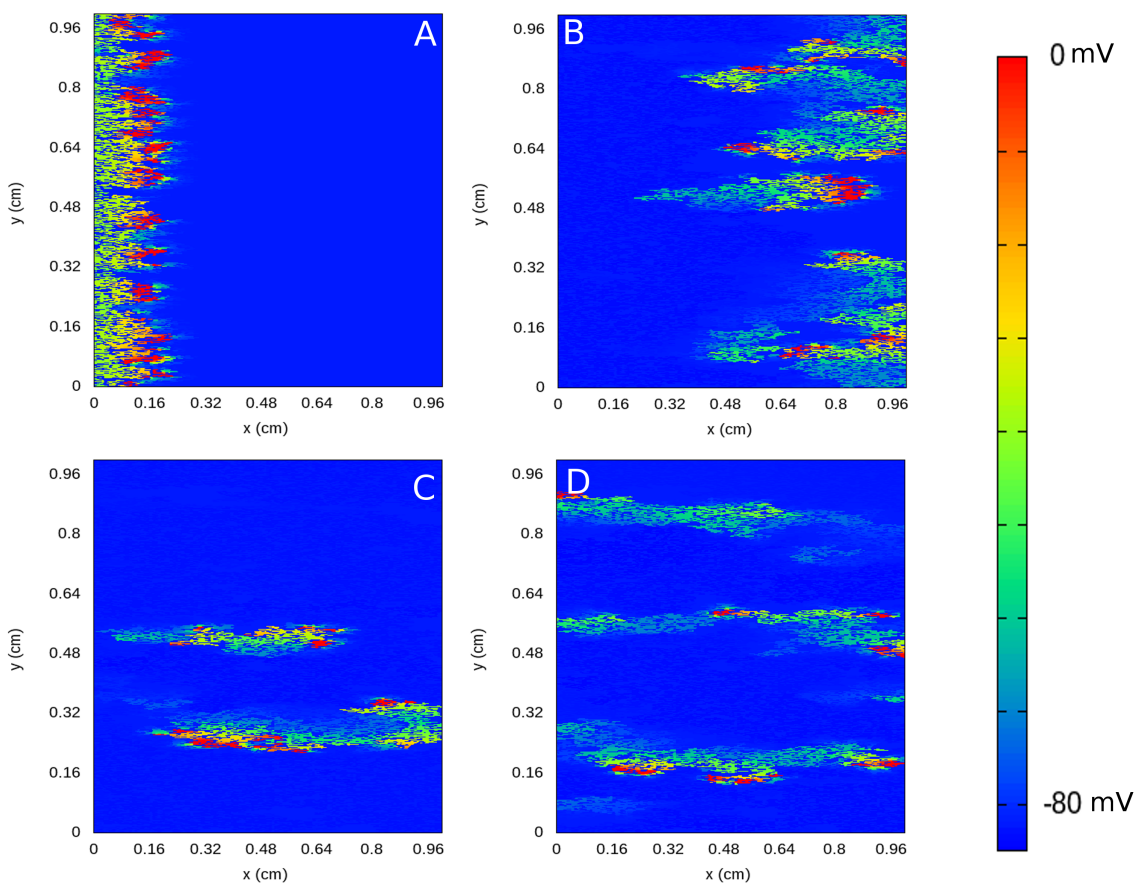

Fig. 4: Sustained reentry inside a fibrotic tissue. Four snapshots showing the transmembrane voltage at times $10 \mathrm{~ms}(\mathrm{~A}), 80 \mathrm{~ms}$ (B), $200 \mathrm{~ms}(\mathrm{C})$, and 360 $m s(\mathrm{D})$. 


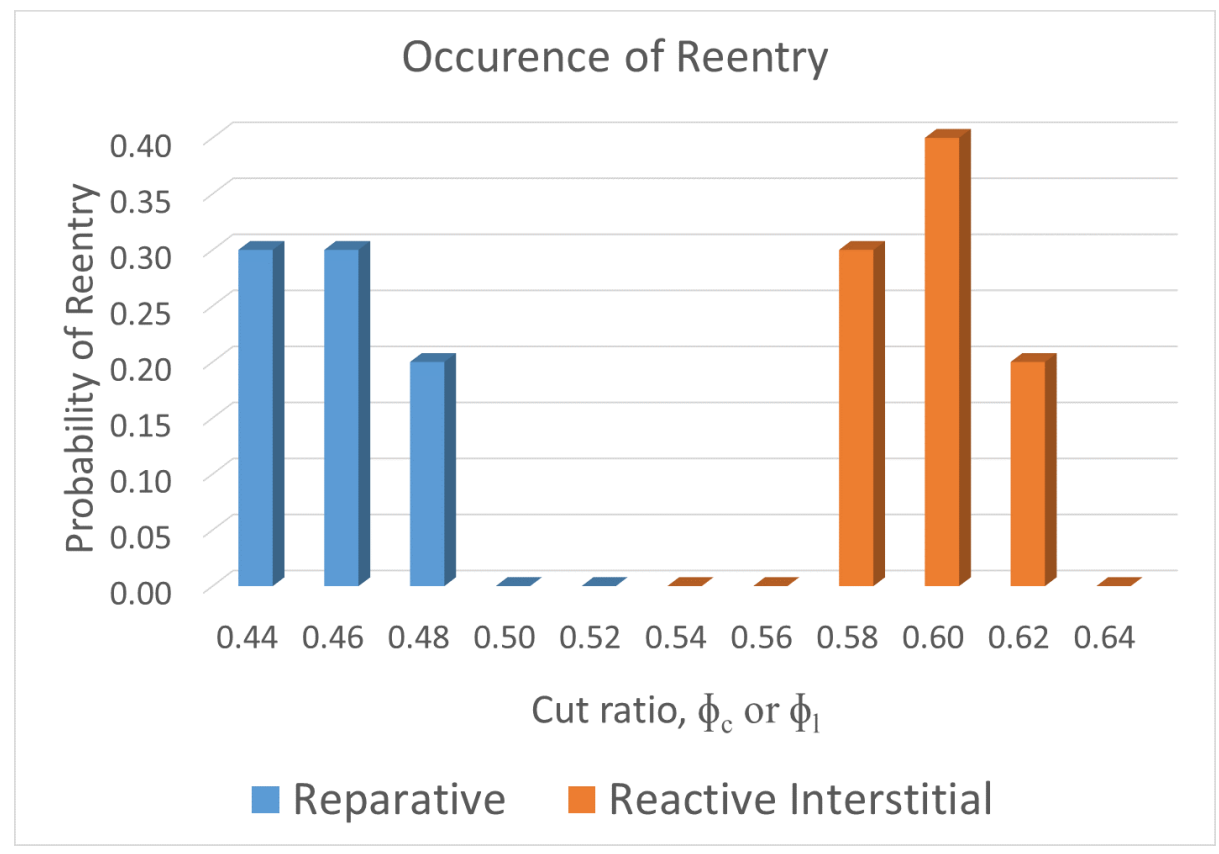

Fig. 5: Probability of reentry for the two cases of fibrosis: reparative vs. reactive interstitial fibrosis. For reactive interstitial fibrosis, $\phi_{l}$ is the percentage of disconnection between cells. For reparative fibrosis, $\phi_{c}$ is the percentage of removed cells.

tial. For both cases the probability of reentry depends on the fraction of removed cells $\left(\phi_{c}\right.$, for the case of reparative fibrosis) or of removed connections or links $\left(\phi_{l}\right.$, for the case of reactive interstitial fibrosis). For both types of fibrosis reentry can appear with probabilities higher than 30\%. However, the probability distribution for the case of reactive interstitial fibrosis is shifted to the right, in terms of $\phi$. Therefore, to become an ectopic pacemaker a microfibrosis region with pure reactive interstitial needs higher values of disconnection between cells $\left(\phi_{l}\right.$ around $\left.60 \%\right)$ than in a pure reparative fibrosis case $\left(\phi_{c}\right.$ around $\left.45 \%\right)$. These values are qualitative near the percolation values computed before in [1] for the case of regular and isotropic tissue where each cell connects to six neighboring cells.

\section{Conclusions}

In this paper we have extended our previous works to evaluate how two different types of fibrosis, reactive interstitial and reparative fibrosis, may support the generation of reentry inside a microfibrosis tissue. We have shown that in both types of fibrosis after the traveling wave enters the microfibrosis region propagation is fractionated and its zig-zag patterns supports sustained reentries. In 
turn, such reentries will continuously try to generate ectopic beats whenever it touches the border of the microfibrosis region. Therefore, fibrosis is shown to be a sufficient substrate for the generation of ectopic pacemakers in diseased cardiac tissues. Although our simulation results supported the thesis that both types of fibrosis can lead to reentries, we have observed that the probability of reentries differs quantitatively for the two different types of fibrosis. To become an ectopic pacemaker a microfibrosis region with pure reactive interstitial needed higher values of disconnection between cells $\left(\phi_{l}\right.$ around 60\%) than in a pure reparative fibrosis case ( $\phi_{c}$ around $45 \%$ ).

In addition, we have also implemented and tested for the first time the new Uniformization numerical method for simulations of cardiac tissue. With the new Uniformization numerical method cardiac tissue simulations were near 20fold faster than when using classical methods such as Euler and Rush-Larsen. Therefore, this was an essential technique that allowed fast execution of over a thousand of simulations.

\section{Acknowledgments}

This work was partially funded by Brazilian Science without Borders, CNPq, Capes, Fapemig, UFJF and Finep; Geman DFG project SFB 910; and MINECO Spain under Ramon y Cajal program RYC-2012-11265.

\section{References}

1. Alonso, S., Bär, M.: Reentry near the percolation threshold in a heterogeneous discrete model for cardiac tissue. Physical review letters 110(15), 158101 (2013)

2. Alonso, S., Sagués, F., Mikhailov, A.S.: Taming winfree turbulence of scroll waves in excitable media. Science 299(5613), 1722-1725 (2003)

3. Balay, S., Abhyankar, S., Adams, M., Brown, J., Brune, P., Buschelman, K., Eijkhout, V., Gropp, W., Kaushik, D., Knepley, M., et al.: Petsc users manual revision 3.5. Tech. rep., Argonne National Laboratory (ANL) (2014)

4. Bär, M., Eiswirth, M.: Turbulence due to spiral breakup in a continuous excitable medium. Physical Review E 48(3), R1635 (1993)

5. Gouvêa de Barros, B., Sachetto Oliveira, R., Meira, W., Lobosco, M., Weber dos Santos, R.: Simulations of complex and microscopic models of cardiac electrophysiology powered by multi-gpu platforms. Computational and mathematical methods in medicine 2012 (2012)

6. de Barros, B.G., dos Santos, R.W., Lobosco, M., Alonso, S.: Simulation of ectopic pacemakers in the heart: multiple ectopic beats generated by reentry inside fibrotic regions. BioMed Research International 2015 (2015)

7. Bondarenko, V., Szigeti, G., Bett, G., Kim, S., Rasmusson, R.: Computer model of action potential of mouse ventricular myocytes. American Journal of Physiology Heart and Circulatory Physiology 287, H1378-H1403 (2004)

8. Dos Santos, R.W., Kosch, O., Steinhoff, U., Bauer, S., Trahms, L., Koch, H.: Mcg to ecg source differences: measurements and a two-dimensional computer model study. Journal of electrocardiology 37, 123-127 (2004) 
9. Finet, J.E., Rosenbaum, D.S., Donahue, J.K.: Information learned from animal models of atrial fibrillation. Cardiology Clinics 27(1), 45 - 54 (2009), atrial Fibrillation

10. Groop, W., Lusk, E.: User's guide for mpich, a portable implementation of MPI. Tech. rep., Argonne National Laboratory (1994)

11. Hodgkin, A.L., Huxley, A.F.: A quantitative description of membrane current and its application to conduction in nerve. Journal of Phisiology 117, 500-544 (1952)

12. Hubbard, M.L., Henriquez, C.S.: A microstructural model of reentry arising from focal breakthrough at sites of source-load mismatch in a central region of slow conduction. American Journal of Physiology-Heart and Circulatory Physiology 306(9), H1341-H1352 (2014)

13. Jensen, A.: Markoff chains as aid in the study of markoff processes. Skandinavisk Aktuarietidskrift 36, 87-91 (1953)

14. Laurent, G., Moe, G., Hu, X., Leong-Poi, H., Connelly, K.A., So, P.P.S., Ramadeen, A., Doumanovskaia, L., Konig, A., Trogadis, J., Courtman, D., Strauss, B., Dorian, P.: Experimental studies of atrial fibrillation: a comparison of two pacing models. American Journal of Physiology - Heart and Circulatory Physiology 294(3), H1206H1215 (2008)

15. Moreira Gomes, J., Alvarenga, A., Silva Campos, R., Rocha, B., Couto da Silva, A., Weber dos Santos, R.: Uniformization method for solving cardiac electrophysiology models based on the markov-chain formulation. IEEE Trans. on Biomed. Eng. 62(2), 600-608 (Feb 2015)

16. Platonov, P.G., Mitrofanova, L.B., Orshanskaya, V., Ho, S.Y.: Structural abnormalities in atrial walls are associated with presence and persistency of atrial fibrillation but not with age. Journal of the American College of Cardiology 58(21), $2225-2232(2011)$

17. Reibman, A., Trivedi, K.: Numerical transient analysis of markov models. Comput. Opns Res. 15(1), 19-36 (1988)

18. Rush, S., Larsen, H.: A practical algorithm for solving dynamic membrane equations. Biomedical Engineering, IEEE Transactions on (4), 389-392 (1978)

19. Rush, S., Larsen, H.: A practical algorithm for solving dynamic membrane equations. IEEE Transactions on Biomedical Engineering 25(4), 389-392 (1978)

20. dos Santos, R., Campos, F., Neumann, L., A., N., Giles, W.: ATX-II effects on the apparent location of $\mathrm{m}$ cells in a computational human left ventricular wedge. Journal of Cardiovascular Electrophysiology 17, S86-S95 (2006)

21. Silver, M.A., Pick, R., Brilla, C.G., Jalil, J.E., Janicki, J.S., Weber, K.T.: Reactive and reparative fibrillar collagen remodelling in the hypertrophied rat left ventricle: two experimental models of myocardial fibrosis. Cardiovascular Research 24(9), 741-747 (1990)

22. Sundnes, J.: Computing the electrical activity in the heart. Springer Verlag (2006)

23. Sundnes, J., Artebrant, R., Skavhaug, O., Tveito, A.: A second-order algorithm for solving dynamic cell membrane equations. IEEE Transactions on Biomedical Engineering 56, 2546-2548 (2009)

24. Tobon, C., Ruiz-Villa, C.A., Heidenreich, E., Romero, L., Hornero, F., Saiz, J.: A three-dimensional human atrial model with fiber orientation. electrograms and arrhythmic activation patterns relationship. PLoS ONE 8(2), e50883 (02 2013)

25. Voigt, N., Dobrev, D.: Cellular and molecular correlates of ectopic activity in patients with atrial fibrillation. Europace 14(suppl 5), v97-v105 (2012)

26. Weiss, J.N., Karma, A., Shiferaw, Y., Chen, P.S., Garfinkel, A., Qu, Z.: From pulsus to pulseless the saga of cardiac alternans. Circulation Research 98(10), 1244-1253 (2006) 